\title{
Statin use decreases the risk of cholangiocarcinoma: A meta-analysis
}

\author{
Gaurav NEPAL*
}

Department of Internal Medicine, Tribhuvan University Institute of Medicine, Kathmandu, Nepal

Introduction: Statins are widely prescribed both for primary and secondary prevention of cardiovascular diseases. Preclinical studies indicate that statins have anticancer properties. Epidemiological studies have shown that statin use is associated with decreased risks of various cancer and cancer related mortality. We performed a meta-analysis of all existing studies investigating the association between statin use and the risk of developing cholangiocarcinoma (CCA), which to the best of our knowledge is the first meta-analysis on this issue.

Methods: A comprehensive literature search for articles and abstracts published up to June 2020 was carried out. For inclusion, studies had to report odds ratio (OR), relative risk, or hazard ratio, with 95\% confidence interval (CI). Pooled adjusted ORs with corresponding 95\% CIs were calculated using random effects model. Publication bias was assessed through Egger's test and Begg test. Heterogeneity was accessed by means of the I2 value.

Results: Five observational studies were included in our analysis, with 8,450 CCA subjects and 978,008 healthy controls. Administration of statins significantly reduced the incidence of CCA (OR $=0.79,95 \%$ CI: $0.73-0.86, p=0.0001)$. No heterogeneity was found in the study $\left(\mathrm{I}^{2}=46 \%, p=0.12\right)$. No evidence of publication bias was observed in this meta-analysis.

Conclusions: Our study shows statistically significant association between the use of statins and 0.79-fold decreased risk of CCA. 